\title{
Inductive and supramolecular effects in 2-Amino-N-(2-Hydroxyphenyl)-Benzamide
}

Joseph P. Haller ${ }^{1}$, Warunee Thaenghin ${ }^{1}$, Wannipa Un-chai ${ }^{1}$, Kenneth J. Haller ${ }^{1}$

${ }^{1}$ School Of Chemistry, Suranaree University Of Technology, Nakhon Ratchasima, Thailand

E-mail: pinsanoi@gmail.com

The structure of 2-amino- $\mathrm{N}$-(2-hydroxyphenyl)-benzamide consists of an aniline ring and a phenol ring bridged by an amide group. The molecular structural analysis shows several interesting aspects. The three $\mathrm{C}-\mathrm{C}$ bond distances adjacent to the ortho amide carbonyl group and the amino group of the substituted aniline ring are longer than the normal $\mathrm{C}(\mathrm{ar})-\mathrm{C}(\mathrm{ar})$ bond (bond order < 1.5) [1] because of the electron withdrawing nature of the substituents. In contrast the $\mathrm{C}-\mathrm{C}$ bond distances in the substituted phenol ring are more uniform and of normal $\mathrm{C}(\mathrm{ar})-\mathrm{C}(\mathrm{ar})$ bond distance because of the compensating nucleophilic and electrophilic effects of the ortho $\mathrm{N}$-amide and hydroxyl groups, respectively. The average $\mathrm{C}(\mathrm{ar})-\mathrm{C}(\mathrm{ar})$ bond distance in the aniline ring, $1.392 \AA$, is the same as the spectroscopic distance in benzene (average bond order $=1.5$ ). The amide nitrogen atom is intermediate between sp2 and sp3 hybridization with electron density delocalized through out the amide ligand and to a lesser extent to both aryl rings; therefore, making the amide nitrogen nonplanar. The molecule is illustrated below, viewed perpendicular to and parallel to the aromatic planes.

The molecule contains four strong hydrogen bond donor sites which are involved in strong $\mathrm{N}-\mathrm{H} \bullet \bullet \bullet \mathrm{O}$ and $\mathrm{O}-\mathrm{H} \bullet \bullet \bullet \mathrm{O}$ hydrogen bond interactions. Three of the strong hydrogen bonds are intramolecular, reinforcing the already nearly planar geometry of the extensively delocalized molecule, while the fourth is intermolecular and connects adjacent molecules into parallel head-to-tail infinite chain structures extending through the crystal in the [0 10 l direction. The one-dimensional chains are interconnected by $\mathrm{C}-\mathrm{H} \bullet \bullet \bullet$ edge-to-face phenyl-phenyl type interactions arranged in a herring bone pattern and by face-to-face $\mathrm{C}-\mathrm{H} \bullet \bullet n$ interactions, thereby creating a three-dimensional hydrogen bonded structure.

1. Burgi, H.-B.; Dunitz, J. D. Structure Correlation, 1998, vol. 2, VCH Publishers, New York, USA.

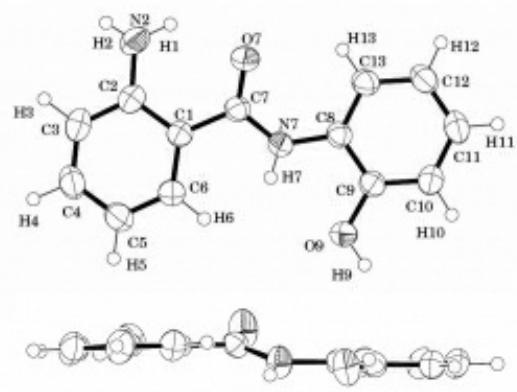

Keywords: inductive effects, supramolecular structure, hydrogen bonding 